\title{
HALO PROPERTIES AND SECULAR EVOLUTION IN BARRED GALAXIES
}

\author{
E. Athanassoula \\ Observatoire de Marseille Provence, 2 place Le Verrier, 13248 Marseille cedex 04, France \\ lia@oamp.fr
}

\begin{abstract}
The halo plays a crucial role in the evolution of barred galaxies. Its near-resonant material absorbs angular momentum emitted from some of the disc particles and helps the bar become stronger. As a result, a bar (oval) forms in the inner parts of the halo of strongly barred disc galaxies. It is thinner in the inner parts (but still considerably fatter than the disc bar) and tends to spherical at larger radii. Its length increases with time, while always staying shorter than the disc bar. It is roughly aligned with the disc bar, which it trails only slightly, and it turns with roughly the same pattern speed. The bi-symmetric component of the halo density continues well outside the halo bar, where it clearly trails behind the disc bar. The length and strength of the disc and halo bars correlate; the former being always much stronger than the latter. If the halo is composed of weakly interacting massive particles, then the formation of the halo bar, by redistributing the matter in the halo and changing its shape, could influence the expected annihilation signal. This is indeed found to be the case if the halo has a core, but not if it has a steep cusp. The formation and evolution of the bar strongly affect the halo orbits. A fraction of them becomes near-resonant, similar to the disc near-resonant orbits at the same resonance, while another fraction becomes chaotic. Finally, a massive and responsive halo makes it harder for a central mass concentration to destroy the disc bar.
\end{abstract}

\section{The role of the halo in the evolution of barred galaxies}

As shown by $N$-body simulations, barred galaxies undergo considerable secular evolution (e.g. Hernquist \& Weinberg 1992; Debattista \& Sellwood 2000; Athanassoula \& Misiriotis 2002 [AM02]; Athanassoula 2002 [A02]; Athanassoula 2003 [A03]; O’Neil \& Dubinski 2003; Valenzuela \& Klypin 2003; Martinez-Valpuesta \& Shlosman 2004; Athanassoula 2005a; MartinezValpuesta, Shlosman \& Heller 2005). The halo plays a major role in this, since it participates in the angular momentum exchange within the galaxy. In an isolated disc galaxy with little or no gas, angular momentum is emitted mainly by near-resonant material in the inner disc (bar region) and absorbed by near- 

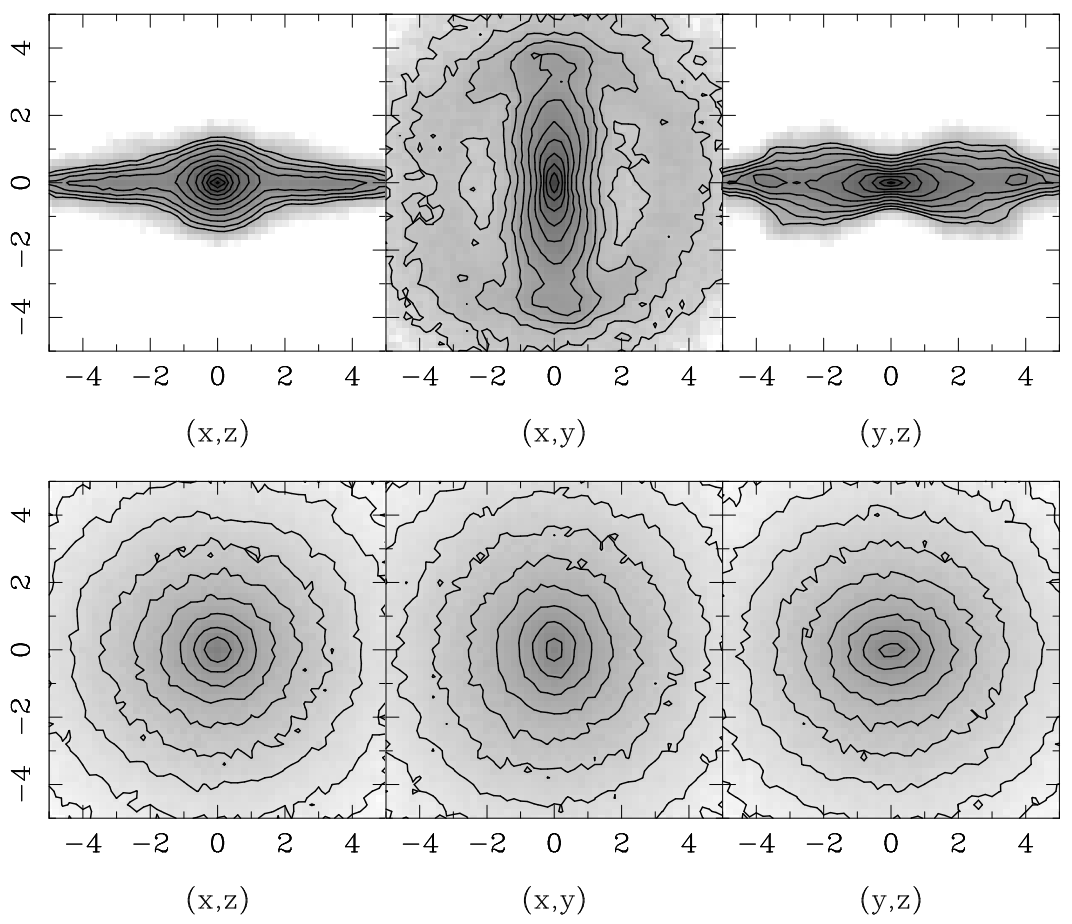

Figure 1. Three orthogonal views of the disc (upper panels) and halo component (lower panels). The central panels are face-on views, while the others are edge-on; side-on for the right panels and end-on for the left ones. Note that the halo does not stay axisymmetric, but forms an oval in its inner parts, which I call the halo bar.

resonant material in the outer disc and in the halo (A02; A03). Since the bar is a negative angular momentum feature (Kalnajs 1971; Lynden-Bell \& Kalnajs 1972), by loosing angular momentum it will grow stronger (A02).

Both for the disc and for the halo, there is more angular momentum gained (or lost) at a given resonance if the density is higher there and if the nearresonant material is colder. So, for equal amounts of mass, the outer disc will absorb more angular momentum than the halo, because it is colder. There is, however, considerably less mass in the outer disc than in the halo, so the role of the halo can be very important, or even predominant (A03). Thus, bars immersed in massive responsive haloes can grow stronger than bars immersed in weaker haloes (AM02; A03) and very much stronger than bars immersed in rigid haloes (A02), since the latter can not, by their formation, absorb any angular momentum.

Since the halo plays such a crucial role in the evolution, it is reasonable to expect that its properties will evolve with time, as do the bar properties. This 

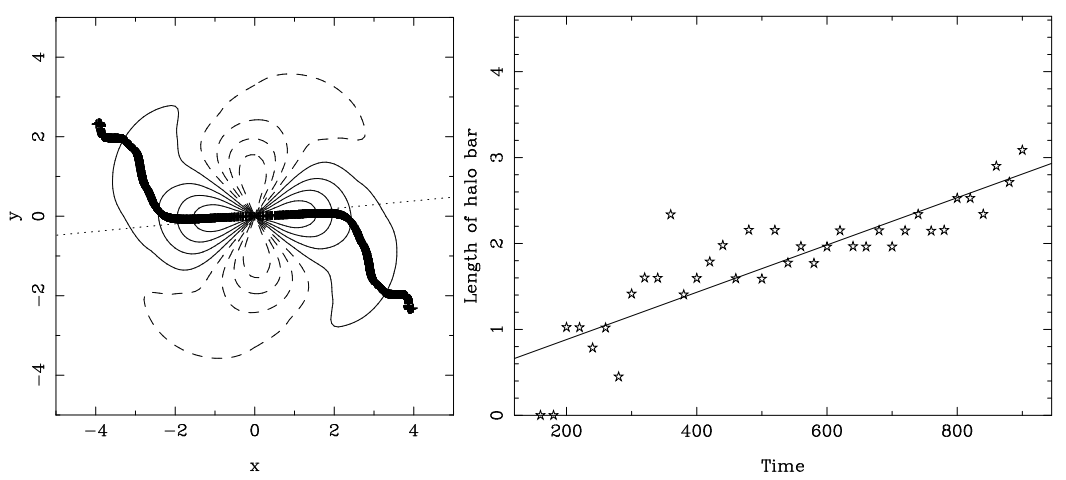

Figure 2. Left panel : Isocontours of the $l=2 m=2$ component of the halo mass distribution on the galaxy equatorial plane. Positive isocontours are given with solid lines and negative ones with dashed lines. The thin dotted line gives the position angle of the disc bar and the thick line shows the phase of the halo bar. The latter is only plotted in regions where the amplitude of the $l=2 m=2$ component is at least equal to $4 \%$ of its maximum amplitude. Right panel : Length of the halo bar as a function of time. The solid line is a least squares fit, to guide the eye.

is indeed the case. Here, I will particularly discuss the formation of an oval in the inner parts of the halo.

\section{The halo bar}

Figure 1, from a simulation similar to those described in AM02 or A03, shows three orthogonal views of the disc (upper panels) and the halo (lower panels) from a simulation at a time well after the bar has formed, i.e. while it is secularly evolving. The disc is strongly barred, and seen side-on (i.e. edgeon, with the line of sight along the bar minor axis) it shows a strong peanut. The evolution of the halo, although less spectacular, is still quite clear. The inner parts have lost their initial spherical symmetry and show a clear prolate deformation, whose principal axes are roughly aligned with those of the disc bar. Such a deformation is seen in all the simulations with a strong bar that I analysed and has also been discussed in Athanassoula (2005b [A05b]) and in Colin, Valenzuela \& Klypin (2005). The axial ratio in the inner parts is roughly 0.7 and increases with radius.

Analysing the halo mass distribution in spherical harmonics, it is possible to get more information on the halo bar. The left panel of Figure 2 shows isocontours of the $l=2 m=2$ component, i.e. of the component that best characterises the halo bar. In the inner parts, the phase of this component seems roughly the same as the phase of the disc bar (dotted line in the figure). In fact, it trails it very slightly, of the order of a couple of degrees so that it is not easy to discern it on the plot. I found similar phase differences at other times and in other 
simulations I analysed. This phase difference increases substantially at larger radii, so that the outer parts of the $l=2 m=2$ component look spiral-like. As can be seen in the right panel of Figure 2, the length of the halo bar increases substantially with time.

\section{Halo geometry and the WIMP annihilation signal}

Athanassoula, Ling and Nezri (2005) studied the impact of halo shape and geometry on the expected WIMP (weakly interacting massive particle) annihilation signal from the galactic center. They find that the asphericity has a strong impact on the annihilation signal when the halo density profile has a core near the center, such as advocated by observations (Bosma 2004 and references therein), but becomes less significant for cuspy profiles, such as advocated by simulations (e.g. Navarro, Frenk \& White 1997; Moore et al. 1999; Diemand et al. 2005), and negligible in the presence of a central spike.

\section{Orbital structure in haloes}

As already mentioned in section 1.1, the halo contains a fair fraction of nearresonant orbits. These are mainly trapped around Lagrangian periodic orbits (see Fig 12 of A05b), or around $x_{1}$-tree ${ }^{1}$ periodic orbits (Fig 11 of A05b).

Halo particles which are near-resonant at a given time are not randomly chosen from the initial halo distribution function. Particles at near-inner Lindblad resonance had initially preferentially smaller cylindrical and spherical radii. They also had preferentially smaller values of $L_{z}$, the $z$ component of the angular momentum. Particles at near-corotation had preferentially initially intermediate cylindrical and spherical radii (i.e. they were not located initially in the innermost or in the outermost regions). They had initially preferentially smaller values of $\left|u_{z}\right|$, the $z$ velocity component, and larger values of $L_{z}$ than average. To summarise, we can say that the halo particles that will become near-resonant had initially properties similar to those of the disc particles.

An important property for the evolution of the halo, and in general of the galaxy, is the fraction of chaotic orbits in its population. The most straightforward way of measuring this in simulations is to calculate the complexity of each orbit, as introduced by Kandrup, Eckstein \& Bradley (1997). As shown by these authors, the complexity correlates well with the short term Lyapounov exponents, often used to measure chaos. Of course, the exact value of the fraction of chaotic orbits depends on where one sets the dividing line between a regular and a chaotic orbit. A05b calculated this fraction for different such threshold values and showed that it increases with the strength of the disc bar and that in a strongly barred galaxy as much as a quarter of the halo orbits can be chaotic. 


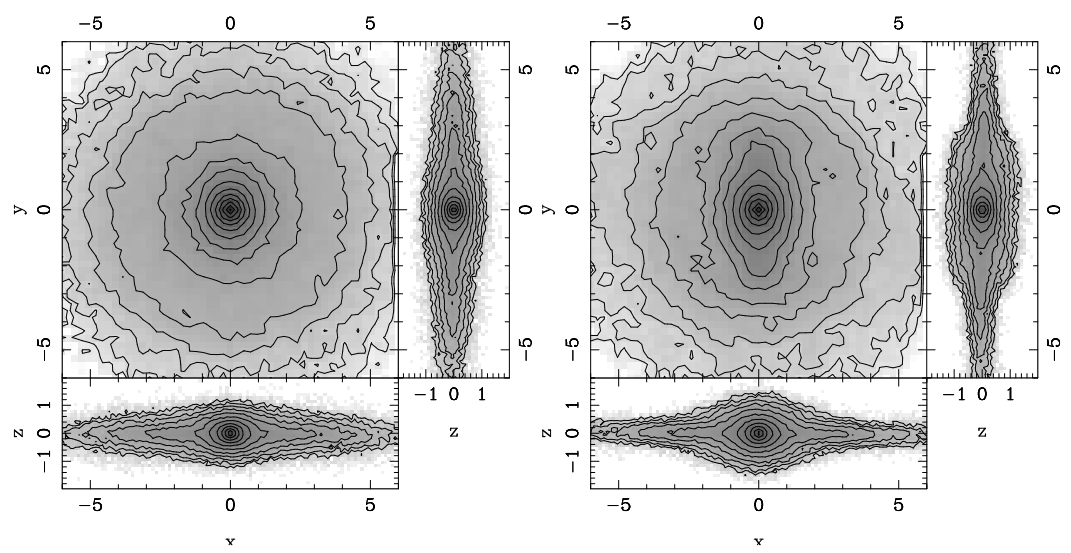

Figure 3. Density distribution in the disc of a barred galaxy after the introduction of a CMC Left panel : MD-type model. Right panel : MH-type model.

\section{Black holes and central mass concentrations in barred galaxies with live haloes}

As shown both by periodic orbit calculations (Hasan \& Norman 1990; Hasan, Pfenniger \& Norman 1993) and by $N$-body simulations (Shen \& Sellwood 2004 and references therein) a central mass concentration (CMC) affects the stability of $\mathrm{x}_{1}$ orbits, making them unstable and incapable of sustaining the bar. Thus, provided the CMC is sufficiently massive and/or sufficiently centrally concentrated, it can destroy the bar, or at least considerably lower its amplitude. These simulations, however, use a rigid halo and thus are not fullyself-consistent.

Seen the important role that the response of the halo can play on the evolution of barred galaxies, Athanassoula, Lambert \& Dehnen (2005) revisited this problem, now using a live halo, and I will here summarise some of their results. They find that the effect of the CMC depends drastically on the model. This is also illustrated in Figure 3, which compares the effect of a given CMC on two different barred galaxy models. The difference is quite important. In the model on the left (MD-type, according to the definition of AM02) the CMC totally destroyed the bar. But in the model on the right (MH-type) the same $\mathrm{CMC}$ only decreased the bar strength, albeit substantially. In fact, the lowering of the bar strength is mainly due to a decrease of the bar length and to more axisymmetric innermost parts. The latter can be easily understood since this is the vicinity of the CMC. The CMC also causes an increase of the bar pattern speed in all cases.

The difference between these two models could be due to the role of the halo in the two cases. Indeed, in MH-type models the inner resonances in the 
halo are more populated, so that the halo can absorb more angular momentum, compared to MD-type haloes (A03). This extra angular momentum is taken from the bar and will, as discussed in section 1.1, tend to increase its strength. It will thus work against the CMC, whose effect will thus be lessened. This is indeed what the simulations of Athanassoula et al. (2005) show.

\section{Notes}

1. The $\mathrm{x}_{1}$ orbits are periodic orbits elongated along the bar and closing after one rotation and two radial oscillations (Contopoulos \& Grosbøl 1989). The $\mathrm{x}_{1}$-tree is the 3D extension of the $\mathrm{x}_{1}$ family (Skokos, Patsis

\& Athanassoula 2002) and comprises, except for the $\mathrm{x}_{1}$ family, other 2D and 3D families bifurcating from $\mathrm{x}_{1}$.

\section{References}

Athanassoula, E., 2002, ApJ, 569, L83 (A02).

Athanassoula, E., 2003, MNRAS, 341, 1179 (A03).

Athanassoula, E., 2005a, MNRAS, 358, 1477.

Athanassoula, E., 2005b, in "Nonlinear Dynamics in Astronomy and Physics (In memory of Henry E. Kandrup)", eds. S. T. Gottesman, J.-R. Buchler and M. E. Mahon, Annals of the New York Academy of Sciences, 1045, 168 (A05b).

Athanassoula, E., Lambert, J. C., Dehnen, W., 2005, MNRAS, in press and astro-ph/0507566. Athanassoula, E., Ling, F.-S., Nezri, E., 2005, Phys. Rev. D., in press and astro-ph/0504631.

Athanassoula, E., Misiriotis, A., 2002, MNRAS, 330, 35 (AM02).

Bosma, A., 2004, in "Dark Matter in Galaxies", eds. S. D. Ryder, D. J. Pisano, M. A. Walker and K. C. Freeman, IAU symposium 220, 39.

Colin, P., Valenzuela, O., Klypin, A., 2005, astro-ph/0506627.

Contopoulos, G., Grosbøl, P., 1989, AAR, 1, 261.

Debattista, V. P., Sellwood, J. A., 2000, ApJ, 543, 704.

Diemand, J., Zemp, M., Moore, B., Stadel, J., Carollo, M., 2005, astro-ph/0504215.

Hasan, H., Norman, C., 1990, ApJ, 361, 69.

Hasan, H., Pfenniger, D., Norman, C., 1993, ApJ, 409, 91.

Hernquist, L., Weinberg, M., 1992, ApJ, 400, 80.

Kalnajs, A. J., 1971, ApJ, 166, 275.

Kandrup, H. E., Eckstein, B. L., Bradley, B. O., 1997, AA, 320, 65.

Lynden-Bell, D., Kalnajs, A. J., 1972, MNRAS, 157, 1.

Navarro, J. F., Frenk, C. S., White, S. D. M., 1997, ApJ, 490, 493.

Martinez-Valpuesta, I., Shlosman, I., 2004, ApJ, 613, L29.

Martinez-Valpuesta, I., Shlosman, I., Heller, C., 2005, ApJ, in press and astro-ph/0507219.

Moore, B., Quinn, T., Governato, F., Stadel, J., Lake, G., 1999, MNRAS, 310, 1147.

O'Neill, J. K., Dubinski, J., 2003, MNRAS, 346, 251.

Shen, J., Sellwood, J. A., 2004, ApJ, 604, 614.

Skokos, Ch., Patsis, P. A., Athanassoula, E., 2002, MNRAS, 333, 847.

Valenzuela, O., Klypin, A., 2003, MNRAS, 345, 406. 